\title{
Análisis de la Productividad Académica de Profesores del Área de Ingeniería
}

\author{
Diofanor Acevedo(1), Piedad M. Montero(1) y Marlene Duran ${ }^{(2)}$ \\ (1) Universidad de Cartagena, Facultad de Ingeniería, Programa de Ingeniería de Alimentos, Avenida el \\ Consulado, Calle 30 No. 48-152. Cartagena, Bolívar-Colombia (e-mail: diofanor3000@gmail.com). \\ (2) Universidad de Cartagena, Facultad de Medicina, Facultad de Medicina, Sede Zaragocilla. Cartagena, \\ Bolívar-Colombia.
}

Recibido May. 26, 2015; Aceptado Jul. 29, 2015; Versión final Sep. 23, 2015, Publicado Abr. 2016

\begin{abstract}
Resumen
El objetivo de este trabajo fue analizar la productividad académica y la percepción de calidad en la enseñanza de los profesores de planta de cuatro áreas de ingeniería de una Institución Universitaria en Colombia. La productividad académica en Colombia, se refiere a las publicaciones de artículos científicos, ponencias, premios, patentes, producciones técnicas y dirección de tesis de postgrado. Se analizaron documentos institucionales y se aplicaron dos cuestionarios, uno a profesores y otro a estudiantes. Los datos recolectados fueron estudiados utilizando análisis de varianza $(p<0,05)$. La mayor productividad académica estuvo en Ingeniería de Alimentos, seguida de Ingeniería Química, Ingeniería de Sistemas e Ingeniería Civil. Se encontró que los estudiantes relacionaron la mayor productividad académica de los profesores con la percepción de calidad en la enseñanza en Ingeniería de Alimentos, mientras que en Ingeniería Civil, la calificación de los estudiantes no estuvo relacionada con la productividad académica de los profesores.
\end{abstract}

Palabras clave: productividad académica, ingeniería, investigación, educación, profesores universitarios

\section{Analysis of the Academic Productivity of University Professors in the Area of Engineering}

\begin{abstract}
The aim of this study was to analyze the academic productivity and the perceived teaching quality of tenure track professors of four engineering majors of a University Institution in Colombia. Academic productivity in Colombia refers to scientific articles publications, congress presentations, awards, patents, technical consulting, and graduate thesis advising. Institutional documents were analyzed and two questionnaires, one for teachers and other for students were applied. The data collected were studied using analysis of variance $(p<0,05)$. The highest academic productivity was in Food Engineering, followed by Chemical Engineering, Systems Engineering and Civil Engineering. It was found that most students related academic productivity of professors with the perception of quality in education in Food Engineering, while for Civil Engineering students there was no relation between productivity and quality teaching.
\end{abstract}




\section{INTRODUCCIÓN}

En los últimos años las políticas de investigación en diferentes países han incorporado mecanismos para incentivar lo que llaman la productividad académica; convencidos del papel central que cumple la investigación dentro del desarrollo económico (Narváez y Tovar, 2011; Araneda-Guirriman et al., 2013; Fassbender y Silva, 2014). De allí que las universidades deben concebirse como centros de productividad académica intelectual, teniendo en cuenta la investigación como eje de desarrollo del conocimiento (GarcíaCepero, 2010). La docencia y la investigación junto a las actividades de extensión representan los componentes del trabajo que debe desempeñar todo profesor universitario con miras a elevar la calidad de su formación integral (Bolívar y Bolívar, 2014). Concepción-González y Ramos-González (2015) señalan que en efecto el resultado de las investigaciones ayuda a mejorar la praxis docente de forma permanente, ya que posibilita la actualización constante. Así mismo Zavala-González et al., (2012) indican que los profesores deben ser líderes, por lo cual es necesario repensar las estructuras organizativas de educación superior, para formar una cultura orientada hacia la investigación.

En Colombia, el Decreto 1279 de 2002 es el que rige los ingresos salariales por productividad académicas de profesores universitarios (Gómez-Campo y Celis-Giraldo, 2007; Fassbender y Silva, 2014). Dicho decreto establece que la productividad académica se mide en términos de publicaciones de artículos en revistas indizadas, libros o capítulos resultados de investigaciones, aunque también se le otorga algún peso a criterios menos exigentes como divulgaciones en revistas no indizadas, revisiones bibliográfica, orientaciones de trabajos de maestría y doctorado, presentaciones en eventos científicos con posters o ponencias, u otros trabajos que aporten al mejoramiento de la calidad estudiantil e institucional. Las bonificaciones son reconocimientos monetarios no salariales que se asignan una sola vez a los profesores, mientras que los puntos salariales representan un incremento en los sueldos que se mantiene a lo largo del tiempo (Gómez-Campo y Celis-Giraldo, 2007; Hernández, 2011; Aguilar et al., 2014).

Según el Departamento Administrativo de Ciencia, Tecnología e Innovación en Colombia (Colciencias) las revistas nacionales e internacionales, donde se publican los productos de investigación de los profesores universitarios, se clasifican de acuerdo a su factor de impacto, el cual se refiere al número de veces que es citada la revista por sus respectivos pares en otras publicaciones o artículos (Alzate-Granados et al., 2014). Estos índices de impacto son construidos y desarrollados por Sistemas de Búsqueda e indización de referencias científicas como ISI o SCOPUS. Siendo Alto (más de 4) equivalente al nivel A1, Medianamente alto $(3-4)$ que corresponde a las revistas clasificadas en $A$, mientras que las revistas $B$ y $C$ se ubican con un factor de impacto medio $(1-3)$ y bajo alrededor de 1, respectivamente (Alzate-Granados et al., 2014).

Por otro lado Gómez-Campo y Celis-Giraldo (2007) mencionan que los mecanismos de estímulos, sobre la medición de la productividad académica, se han convertido primordialmente en instrumentos de autocontrol y que han llevado a la reprobación y deterioro de la pedagogía del docente universitario, pues favorecen exclusivamente a los productos de investigación y no la enseñanza de calidad. Así mismo Carrillo-Vargas (2014), Fassbender y Silva (2014) y Uluocha y Mabawonku (2014) indican que la principal característica de tales mecanismos de estímulos por productividad académica es que no dan cuenta de la calidad de los procesos pedagógicos de enseñanza de los profesores universitarios. Por su parte Comas-Rodríguez y Rivera-Morales (2011) analizaron las políticas de estímulos económicos de profesores universitarios y señalaron que la forma en la que se plantearon, han perjudicado la calidad educativa. En esta misma tendencia Amado-Moreno et al., (2013) realizaron un análisis comparativo de la productividad académica entre instituciones de educación superior y revelaron que en estas los profesores dedicaron poco tiempo a la investigación, pues su tiempo de trabajo estuvo más orientado hacia la catedra en los salones de clases. Teniendo en cuenta lo anterior, el objetivo de este trabajo fue analizar la productividad académica y la relación de esta con la percepción de calidad en la enseñanza y pedagogía de los profesores de planta del área de ingeniería de una institución de educación superior en Colombia durante el año 2014.

\section{MATERIALES Y MÉTODOS}

\section{Tipo de estudio y fuentes de información}

Se realizó un estudio de tipo analítico-descriptivo y de corte transversal, similar a lo realizado por CarrilloVargas (2014) y Concepción-González y Ramos-González (2015). Se consultaron documentos institucionales de acceso público, en los cuales se clasificaron los reconocimientos salariales y bonificaciones de los profesores de planta del área de Ingeniería, según los lineamientos del Decreto 1279 (2002) en la institución de educación superior durante el año 2014. Se halló el número de artículos científicos publicados en revistas nacionales e internacionales indizadas y no indizadas, así como los libros y los otros productos de investigación como posters y ponencias realizados en eventos científicos del área de Ingeniería y que fueron validados como reconocimiento salarial a los profesores. Así mismo se consiguió 
información primaria al aplicar dos cuestionarios (ad hoc) diseñados específicamente para esta investigación, similar a lo realizado por García-Cepero, (2010). El primer formato fue aplicado a una muestra profesores y segundo a una muestra de estudiantes que recibieron clases de los profesores seleccionados; con la finalidad de analizar la percepción de calidad de la enseñanza impartida.

\section{Población y muestra}

Se utilizó una combinación de estadística y análisis de caso (López, 2004) se obtuvo una muestra probabilística estratificada correspondiente a 24 profesores, distribuidos de la siguiente manera: 12 de mayor productividad académica, es decir aquellos que publicaron al menos dos productos de investigación tipo artículo en revistas científicas indizadas y 12 de menor productividad académica, correspondientes a aquellos que no publicaron algún producto de investigación durante el periodo analizado. La población total fueron 56 profesores de planta en la respectiva institución de educación superior. Posteriormente utilizando las mismas técnicas estadísticas y estratificación (López, 2004) se seleccionó una muestra de 144 individuos, de una población de 350 estudiantes que durante el transcurso del año 2014 recibieron clases de estos profesores. Ambas muestras se esquematizaron en la Tabla 1; dónde, el Grupo A= Profesores de mayor Productividad Académica; y el Grupo B= Profesores de menor Productividad Académica; Grupo $C=$ Estudiantes que recibieron clases de los profesores del grupo A; y Grupo D= Estudiantes que recibieron clases de los profesores del grupo B.

Tabla 1: Muestra de profesores y estudiantes seleccionados

\begin{tabular}{|c|c|c|c|c|c|c|}
\hline \multirow{2}{*}{ Áreas } & \multicolumn{3}{|c|}{ Profesores } & \multicolumn{3}{c|}{ Estudiantes } \\
\cline { 2 - 8 } & Grupo A & Grupo B & Total & Grupo C & Grupo D & Total \\
\hline Ingeniería de Alimentos & 3 & 3 & 6 & 18 & 18 & 36 \\
\hline Ingeniería Química & 3 & 3 & 6 & 18 & 18 & 36 \\
\hline Ingeniería de Sistemas & 3 & 3 & 6 & 18 & 18 & 36 \\
\hline Ingeniería Civil & 3 & 3 & 6 & 18 & 18 & 36 \\
\hline Total & 12 & 12 & 24 & 72 & 72 & 144 \\
\hline
\end{tabular}

Instrumentos de la Investigación

A la muestra de profesores seleccionados se le aplicó un cuestionario presencial y en forma de test en físico compuesto por un total de 16 preguntas estructuradas. Las dimensiones del cuestionario indagaron respecto al número artículos científicos realizados por los profesores, así como la participación en eventos científicos, la financiación de proyectos de investigación, la orientación de trabajos de grado, la vinculación a grupos de investigación, su clasificación como investigador, así como el nivel de dominio del idioma inglés. Por su parte el cuestionario aplicado a los estudiantes, constó de 12 preguntas estructuradas, compuestas por cuatro dimensiones, que indagaron aspectos referentes a la calidad de enseñanza en las clases, el nivel de cumplimiento y la motivación de los profesores hacia los estudiantes. La calificación impuesta por los estudiantes varió de $1=$ deficiente, $2=$ insuficiente, $3=$ aceptable, $4=$ bueno y $5=$ excelente.

\section{Análisis estadístico de los datos}

Se analizaron los correspondientes indicadores de productividad recopilados, empleando técnicas de estadística descriptiva, así mismo la información y los datos obtenidos con los cuestionarios aplicados a las muestras objeto de estudio, fueron procesados primero en hojas de cálculo del programa MICROSOFT@ EXCEL(C) versión 2013 para Windows@, y posteriormente en el Programa Estadístico para Ciencias Sociales, IBM SPSS@ - versión 19.0. Se agruparon los valores y compararon las medias entre las área de Ingeniería (Civil, Química, Alimentos y Sistemas) utilizando análisis de varianzas (ANOVA de un factor) y la prueba de Fischer con un nivel de significancia del $5 \%(p<0,05)$.

\section{RESULTADOS Y DISCUSIONES}

En la Tabla 2, se esquematiza los productos de investigación de los profesores de planta del área de Ingeniería, durante el año 2014 (enero-diciembre). Se observó que la mayor producción de artículos publicados en revistas categorizadas en A1 y A2, fueron realizados por los profesores de Ingeniería de Alimentos, seguidos por Ingeniería Química, Ingeniería de Sistemas y por último Ingeniería Civil. En el caso de Ingeniería de Alimentos e Ingeniería Química, se resalta un importante número de posters y ponencias realizadas en eventos científicos durante el transcurso del año 2014, registrados en los documentos institucionales consultados de la respectiva institución educativa. 
Tabla 2: Productividad académica de los 56 profesores de planta del área de Ingeniería durante el año 2014

\begin{tabular}{|c|c|c|c|c|c|c|c|c|c|c|}
\hline \multirow[b]{2}{*}{ Áreas } & \multicolumn{5}{|c|}{ Artículos en Revistas } & \multicolumn{4}{|c|}{ Otros productos de Investigación } & \multirow[b]{2}{*}{ Profesores } \\
\hline & A1 & A2 & B & C & No Indizados & Libros & Postrer & Ponencias & $\begin{array}{c}\text { Trabajos de } \\
\text { Maestría dirigidos }\end{array}$ & \\
\hline Ingeniería de Alimentos & 14 & 16 & 2 & 3 & 8 & 2 & 10 & 12 & 0 & 11 \\
\hline Ingeniería Química & 6 & 5 & 4 & 2 & 3 & 0 & 7 & 27 & 0 & 12 \\
\hline Ingeniería de Sistemas & 8 & 0 & 3 & 1 & 6 & 0 & 5 & 5 & 0 & 9 \\
\hline Ingeniería Civil & 4 & 2 & 2 & 0 & 1 & 2 & 3 & 7 & 0 & 24 \\
\hline Total & 32 & 23 & 8 & 6 & 18 & 4 & 25 & 51 & 0 & 56 \\
\hline
\end{tabular}

En la Tabla 3, se detalla la evolución del nivel formativo de los profesores de planta del área de Ingeniería, durante los años 2010, 2011, 2012, 2013 y 2014. Se puede ver un notable incremento en el número de doctores, así mismo una disminución en el número de especialistas. Lo cual indica que el personal docente se ha ido capacitando en estudios superiores, lo que es indispensable para fomentar y fortalecer la investigación y ayudar en la formación de estudiantes de pregrados, especializaciones y maestrías.

Tabla 3: Nivel formativo de los profesores de Ingeniería durante el 2014. Dónde a=primer periodo; b= segundo periodo.

\begin{tabular}{|c|c|c|c|c|c|c|c|c|c|c|}
\hline Años & \multicolumn{2}{|c|}{2010} & \multicolumn{2}{|c|}{2011} & \multicolumn{2}{c|}{2012} & \multicolumn{2}{c|}{2013} & \multicolumn{2}{c|}{2014} \\
\hline Nivel de Formación & $\mathrm{a}$ & $\mathrm{b}$ & $\mathrm{a}$ & $\mathrm{b}$ & $\mathrm{a}$ & $\mathrm{b}$ & $\mathrm{a}$ & $\mathrm{b}$ & $\mathrm{a}$ & $\mathrm{b}$ \\
\hline Doctorado & 6 & 10 & 10 & 11 & 12 & 11 & 13 & 14 & 15 & 17 \\
\hline Especialización & 20 & 19 & 18 & 19 & 16 & 15 & 14 & 12 & 14 & 10 \\
\hline Maestría & 20 & 18 & 22 & 23 & 23 & 24 & 24 & 27 & 24 & 26 \\
\hline Profesional & 4 & 4 & 4 & 6 & 4 & 4 & 4 & 3 & 3 & 3 \\
\hline Total & 50 & 51 & 54 & 59 & 55 & 54 & 55 & 56 & 56 & 56 \\
\hline
\end{tabular}

Al analizar en detalle la formación académica de los profesores de planta del área de Ingeniería durante el segundo semestre del 2014. Se observó el mayor número de doctores en Ingeniería Química con 50,0\% seguido por Ingeniería de Alimentos con 45,4\%, Ingeniería de Sistemas 22,2\%, e Ingeniería Civil con $16,6 \%$. Mientras que en nivel de maestría sobresalió el área de Civil con nueve, seguido por Sistemas con siete, Alimentos y Química con cinco.

En la Figura 1, se detallan los resultados obtenidos de la parte uno (preguntas 1 a la 4), del cuestionario aplicado a la muestra de profesores de planta del área de Ingeniería, de la institución de educación superior. Los profesores de mayor productividad (grupo A) indicaron poseer estrategias claras al momento de escribir un artículo científico, mientras que un gran porcentaje en los de menor productividad (grupo B) señaló no poseer estrategias para realizar manuscritos científicos; estos resultados fueron similares a los obtenidos por García-Cepero (2010) quienes indican que dentro de las estrategias más importantes que deben ser utilizadas por los profesores para escribir artículos científicos, encontramos la buena redacción, manejo de bases de datos científicas, lectura de texto en inglés, discusión de resultados obtenidos de proyectos de investigación y manejo del diseño experimental y estadístico en las investigaciones. Complementando los resultados hallados en este trabajo, Concepción-González y Ramos-González (2015) indicaron que si los profesores universitarios carecen de estrategias de redacción, y si no conocen los requisitos y normas para realizar una publicación, es muy difícil que realicen artículos, libros, y demás productos de investigaciones.

Por su parte, cuando se les preguntó si estuvieron de acuerdo con los tipos de estímulos ofrecidos por productividad académica enmarcados en el Decreto 1279 (2002) (Fassbender y Silva, 2014) los del grupo A indicaron estar a favor, mientras que los del grupo B, gran cantidad indicó no estar de acuerdo. Lo que posiblemente puede ser explicado por lo discutido por Gómez-Campo y Celis-Giraldo (2007) quienes señalaron que aunque a los profesores de planta en las universidades públicas colombianas, se les incentive salarialmente por los productos de investigación, a estos no se les ofrece plenas y excelentes condiciones laborales que promueva la investigación como eje fundamental del conocimiento. Así mismo estos autores Gómez-Campo y Celis-Giraldo (2007) y Fassbender y Silva (2014) afirmaron que en Colombia dado el tipo de estímulos definidos en el Decreto 1279 es muy probable que los profesores de planta en las instituciones de educación superior públicas, tiendan a valorar más la investigación que esmerarse por ser buenos profesores; esta evidencia demuestra que dicha reglamentación busca promover más los productos de investigación, que la docencia de calidad. La responsabilidad de reconocer y estimular la buena enseñanza es exclusiva de las universidades, por lo que están en la obligación de definir y desarrollar criterios que así lo permitan (Araneda-Guirriman et al., 2013; Aguilar et al., 2014). 


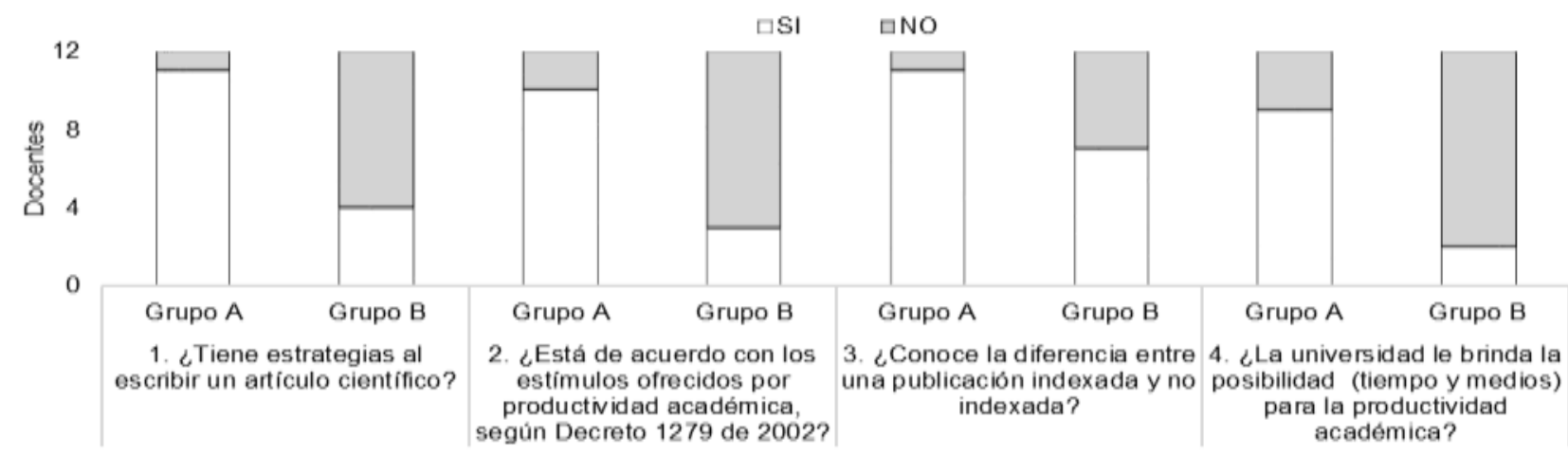

Fig. 1: Respuestas parte uno del cuestionario aplicado a la muestra de profesores de planta del área de Ingeniería de la institución de educación superior, (preguntas 1 a 4). Grupo A: Mayor Productividad. Grupo B: Menor Productividad

En general ambos grupos de profesores reportaron que conocían las diferencias entre las publicaciones indizadas y no indizadas. Lo cual fue similar a lo reportado por Amado-Moreno et al., (2013) en su investigación sobre la productividad académica en las instituciones públicas tecnológicas mexicanas, y a lo hallado por García-Cepero (2010) en el estudio de productividad académica de profesores universitarios. Ambos autores reportaron dentro de sus discusiones que cuando un artículo está indizado, quiere decir que ha pasado por un proceso de selección y análisis por parte de instituciones o empresas documentarias que realizan ese trabajo y para determinar si una revista ingresa a un índice, se toman en cuenta ciertos criterios de calidad, que pueden ser agrupados en: a) calidad del contenido de la investigación; b) características técnicas o formales; c) uso por parte de la comunidad científica (o impacto).

En la Figura 2, se detallan los resultados obtenidos de la parte uno (preguntas 5 a la 8). Los profesores del grupo A, indicaron que la institución de educación superior le ha brindado la oportunidad de capacitarse en su área de formación, ya sea en estudios de maestrías y doctorados. Se resalta que ambos grupos de profesores señalaron desconocer que la institución les brindó la posibilidad de traducir sus artículos para publicación en inglés.

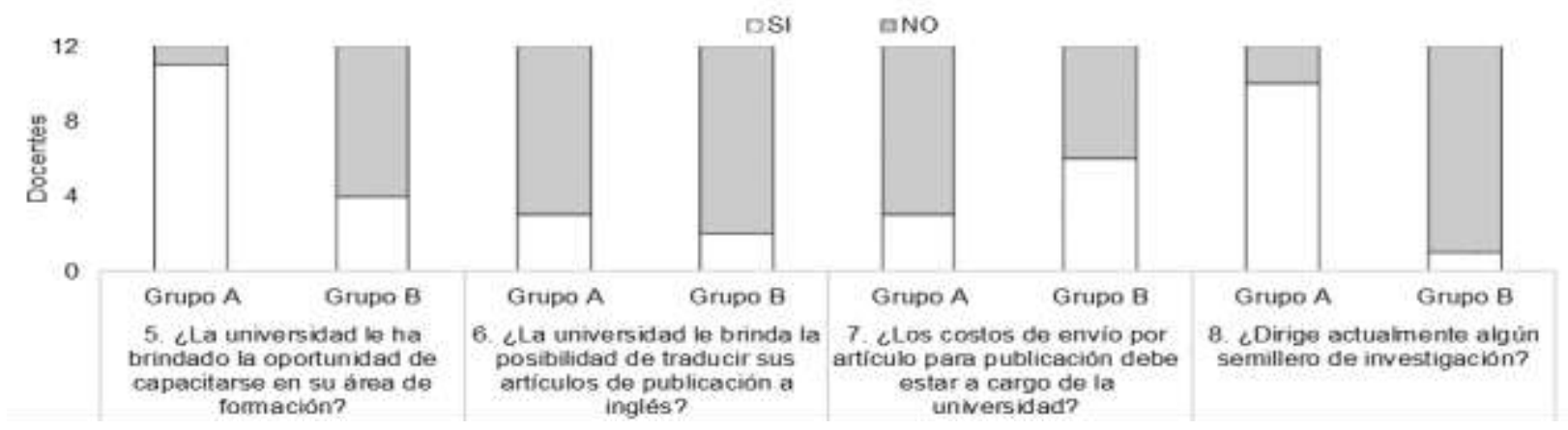

Fig. 2: Respuestas parte uno del cuestionario aplicado a la muestra de profesores de planta del área de Ingeniería de la institución de educación superior (preguntas 5 a la 8) Grupo A: Mayor Productividad. Grupo B: Menor Productividad

En cuanto a los costos de envío de artículos para publicación, ambos grupos de profesores indicaron estar de acuerdo que la institución debe asumir dichos rubros, es decir que si debe ocuparse de estos costos económicos. Los profesores de mayor productividad académica indicaron dirigir los semilleros de investigación, mientras que los de menor productividad revelaron que no tenían a su cargo estudiantes de pregrado en formación investigativa. Estos resultados refuerzan lo argumentado por Bolívar y Bolívar (2014) quienes señalaron que son los profesores de mayor nivel formativo en estudios doctorales y postdoctorales, los que deben estar a cargo de los grupos y semilleros de investigación, ya que estos al estar en contacto con el conocimiento actualizado permitirán ampliar la visión investigativa de los estudiantes.

En la Tabla 4, se presentan las respuestas de la parte dos del cuestionario aplicado a los profesores (preguntas 9 a la 16). Cuando a los profesores se les preguntó sobre el número de veces que ingresaron a bases de datos científicas, la mayoría del grupo A, contestaron que visitaron dichas páginas entre 7 y 12 veces a la semana. Mientras que los del grupo $B$, señalaron que muy poco accedían a dichas bases de datos y revistas científicas de Ingeniería; lo cual indica que en estas áreas los profesores no se interesaron por buscar información científica para discutir resultados de investigación. Se observó que en general los profesores de mayor productividad tuvieron al menos un proyecto de investigación aprobado y financiado por la institución de educación superior, mientras que los del grupo B, la mayoría señalaron no tener proyectos de investigación financiados. Tendencia similar fue observada cuando se preguntó referente a los 
proyectos financiados por entidades externas. Los profesores del grupo A, fueron los que mayor número de estudiantes investigadores tuvieron a su cargo. Los profesores del grupo B, en su mayoría no presentaron categorización como investigadores, según Colciencias. Mientras que en los de mayor productividad la clasificación osciló entre Junior y Asociados.

Tabla 4: Respuestas parte dos del cuestionario aplicado a la muestra de profesores de planta del área de la Institución de educación superior. Grupo A: Mayor Productividad. Grupo B: Menor Productividad

\begin{tabular}{|c|c|c|}
\hline Preguntas/Opciones. & \multicolumn{2}{|c|}{ Profesores $(n=24)$} \\
\hline 9. ¿Cuantos artículos ha publicado en el último año en revistas indizadas? & Grupo A & Grupo B \\
\hline a) ninguno & 0 & 8 \\
\hline b) 1 a 3 & 2 & 4 \\
\hline c) 4 a 6 & 7 & 0 \\
\hline d) 7 a 10 & 3 & 0 \\
\hline 10. ¿A cuántos eventos de investigación ha asistido con poster o ponencia el último año? & Grupo A & Grupo B \\
\hline a) ninguno & 0 & 6 \\
\hline b) 1 a 2 & 3 & 4 \\
\hline c) 3 a 4 & 4 & 2 \\
\hline d) 5 a 6 & 5 & 0 \\
\hline 11. ¿Cuantas veces ingresa usted al día a bases de datos científicas? & Grupo A & Grupo B \\
\hline a) ninguna & 0 & 6 \\
\hline b) 1 a 6 & 2 & 5 \\
\hline c) 7 a 12 & 6 & 1 \\
\hline d) 13 a 20 & 4 & 0 \\
\hline 12. ¿Cuantos proyectos aprobados y financiados por la universidad o entidades externas en el 2014 ? & Grupo A & Grupo B \\
\hline a) ninguna & 0 & 8 \\
\hline b) 1 & 9 & 4 \\
\hline c) 2 & 3 & 0 \\
\hline d) 3 & 0 & 0 \\
\hline 13. ¿Cuantos jóvenes investigadores dirigió en el último año? & Grupo A & Grupo B \\
\hline a) ninguna & 1 & 8 \\
\hline b) 1 & 9 & 3 \\
\hline c) 2 & 2 & 1 \\
\hline d) 3 & 0 & 0 \\
\hline 14. ¿Cuál es su clasificación como investigador? & Grupo A & Grupo B \\
\hline a) No Tiene & 0 & 7 \\
\hline b) Junior & 5 & 5 \\
\hline c) Asociado & 4 & 0 \\
\hline d) Sénior & 3 & 0 \\
\hline 15. ¿En qué nivel de inglés considera que se encuentra actualmente? & Grupo A & Grupo B \\
\hline a) A1 & 1 & 4 \\
\hline b) A2 & 3 & 3 \\
\hline c) B1 & 5 & 5 \\
\hline d) B2 & 3 & 0 \\
\hline 16. ¿Cuál es la clasificación del grupo de investigación al que está vinculado? & Grupo A & Grupo B \\
\hline a) No tiene & 0 & 5 \\
\hline b) $\mathrm{D}$ & 3 & 4 \\
\hline c) $\mathrm{C}$ & 6 & 3 \\
\hline d) $\mathrm{B}$ & 2 & 0 \\
\hline e) A & 1 & 0 \\
\hline
\end{tabular}

Es importante resaltar que en Colombia la clasificación en los niveles de inglés de los investigadores y profesionales, son certificados de acuerdo al marco común de referencia europeo (Desde A1 y A2 el nivel básico, B1 y B2 intermedio; hasta C1 y C2 avanzado). Por lo cual al analizar las respuestas entregadas por los profesores, se encontró que ambos grupos indicaron que su dominio del inglés osciló entre A2 y B2, lo cual indicó un nivel intermedio, es decir que los profesores según esta clasificación fueron capaces de comprender frases y expresiones de uso frecuente en inglés relacionadas con sus respectivas áreas de experiencia, incluso de carácter técnico siempre que estuviesen dentro de su campo de especialización. Estos resultados fueron similares a los encontrados por Zavala-González et al., (2012) en su trabajos sobre un diagnóstico situacional de la productividad científica de profesores-investigadores universitarios. Dichos autores manifestaron que es indispensable que los profesores en los niveles más altos de formación académica, maestrías, doctorados y postdoctorados, posean habilidades comunicativas con los idiomas, especialmente con el inglés, ya que gran parte de las investigaciones recientes encontradas en bases de datos científicas están en este lenguaje, por tanto si se quiere estar actualizado es necesario aprender dicho idioma.

En el presente trabajo los resultados hallados sobre la baja productividad académica de algunos profesores (grupo B), coinciden con lo señalado por Amado-Moreno et al., (2013) y Carrillo-Vargas (2014) los cuales lo atribuyen a factores como, la poca motivación y escaso tiempo de dedicación a las actividad investigativas; así mismo García-Cepero (2010) encontró que los profesores de planta en universidades estatales dedicaron gran parte de su tiempo a dictar clases en las aulas y escaso tiempo a la investigación, debido 
principalmente a la carga horaria. Por su parte Narváez y Tovar (2011) encontraron que los resultados de la baja productividad académica están también relacionados con la poca exigencia de las autoridades administrativas de los centros educativos, por ello mencionan que es indispensable que los profesores de planta realicen al menos una publicación en revistas internacionales indizadas, para de esta manera estimular el crecimiento en investigación y esto ayude a promover la categoría docente.

Por su parte otros autores como Aguilar et al., (2014) mencionan que aumentar de manera acelerada la productividad académica, sin llevar un control exacto, puede ocasionar que en esencia baje la calidad científica de lo que se quiera publicar. Así mismo Hernández (2011) señala que la publicación científica en el ámbito superior, constituye un indicador importante y una herramienta básica en el desarrollo profesional, por lo que se debe tener en cuenta los atributos que dan cuenta de su calidad, entre ellos la categoría de la revista donde se realiza la publicación, y la extensión de los trabajos. En general en la institución de educación superior analizada, teniendo en cuenta el nivel formativo de los profesores de planta del área de Ingeniería, sigue siendo escaso el número publicaciones en revistas internacionales indizadas. Lo cual coincide con lo encontrado por Lee (2014) quien encontró que dichas publicaciones de profesores de plantas de universidades estatales, en este tipo de revistas internacionales A1, son escasas debido principalmente a que no todos los profesores disponen de los recursos necesarios para su acceso, además estas revista suelen ser más rigurosos en sus arbitrajes, exigiendo resultados novedosos y con un aporte científico y/o social importante. Lo cual coincide con lo mencionado por Uluocha y Mabawonku (2014) quienes analizaron la investigación y productividad académica en universidades de Nigeria y reportaron que los elevados costos económicos de edición que imponen algunas revistas de publicación internacional y de alto impacto, impiden el acceso a profesionales de países subdesarrollados o en vía de desarrollo con recursos limitados, lo que influye en la poca visibilidad de sus resultados de investigación ante la comunidad científica mundial.

En la Tabla 5, se esquematizan las calificaciones realizadas por parte de los estudiantes, a los profesores de planta del área de Ingeniería. En general se encontró que los de mayor productividad académica en los áreas Ingeniería de Alimentos, Química y Sistemas, fueron percibidos por los estudiantes como mejores, en cuanto al nivel de enseñanza, exigencia, cumplimiento, motivación y apoyo con material didáctico en las asignaturas. Mientras que en el área Ingeniería Civil, los alumnos indicaron que se adaptaron mejor a las clases con los profesores del grupo B, lo cual puede ser debido a que dichos profesores combinaron el aprendizaje teórico de las aulas de clases con el saber práctico de las empresas del sector en construcción y asesoría de obras Civiles, en las cuales tuvieron injerencia. En comparación con los profesores de mayor productividad en dicho área que se dedicaron a la investigación.

Tabla 5: Evaluación de los Estudiantes sobre la Calidad de Enseñanza de los Profesores de Planta del área de Ingeniería. Grupo A: profesores de mayor productividad. Grupo B: profesores de menor productividad

\begin{tabular}{|c|c|c|c|c|c|c|c|c|}
\hline $\begin{array}{c}\text { Áreas } \\
\text { Item de Evaluación }\end{array}$ & \multicolumn{2}{|c|}{$\begin{array}{l}\text { Ing. Alimentos } \\
(\mathrm{n}=36)\end{array}$} & \multicolumn{2}{|c|}{$\begin{array}{c}\text { Ing. Química } \\
(\mathrm{n}=36)\end{array}$} & \multicolumn{2}{|c|}{$\begin{array}{l}\text { Ing. Sistemas } \\
(\mathrm{n}=36)\end{array}$} & \multicolumn{2}{|c|}{$\begin{array}{l}\text { Ing. Civil } \\
(\mathrm{n}=36)\end{array}$} \\
\hline Aspectos a evaluar de los Profesores & A & $\mathrm{B}$ & A & B & A & $B$ & $\bar{A}$ & $\mathrm{~B}$ \\
\hline ¿El programa de la materia fue claro? & 3,45 & 2,75 & 3,33 & 2,39 & 3,84 & 1,87 & 2,95 & 3,96 \\
\hline ¿Cómo le pareció la metodología de trabajo? & 4,09 & 2,44 & 4,43 & 4,43 & 3,79 & 3,88 & 3,97 & 4,11 \\
\hline ¿El docente dominó el contenido de la asignatura? & 3,57 & 3,65 & 2,98 & 3,75 & 2,29 & 3,94 & 3,55 & 3,19 \\
\hline ¿El docente promovió la investigación? & 4,82 & 3,49 & 3,93 & 2,67 & 2,26 & 2,27 & 3,18 & 3,33 \\
\hline ¿El docente exigió el cumplimiento y entrega de trabajos? & 4,32 & 3,35 & 2,74 & 2,49 & 2,99 & 2,92 & 3,06 & 4,86 \\
\hline ¿El docente abordó todos los contenidos temáticos programados? & 4,83 & 2,84 & 2,67 & 2,84 & 2,95 & 3,82 & 2,76 & 4,33 \\
\hline $\begin{array}{c}\text { ¿El docente aplicó los criterios de evaluación tal como los estableció } \\
\text { e informó oportunamente los resultados? }\end{array}$ & 3,73 & 2,93 & 3,84 & 3,67 & 3,67 & 4,28 & 3,59 & 2,36 \\
\hline ¿El docente mostró interés por el aprendizaje de los alumnos? & 4,43 & 2,77 & 4,73 & 2,94 & 3,94 & 2,45 & 2,19 & 4,76 \\
\hline ¿Es el docente fue abierto al diálogo? & 3,29 & 2,98 & 2,83 & 2,87 & 3,18 & 3,47 & 3,11 & 4,98 \\
\hline ¿Los materiales utilizados apoyaron en el logro del aprendizaje? & 4,32 & 2,96 & 3,39 & 2,98 & 2,76 & 3,28 & 2,12 & 3,97 \\
\hline ¿Las actividades permitieron alcanzar los objetivos del curso? & 3,72 & 3,55 & 2,95 & 2,84 & 3,88 & 2,79 & 3,41 & 4,96 \\
\hline ¿El docente promovió la utilización de herramientas virtuales? & 4,44 & 2,91 & 3,74 & 2,79 & 2,76 & 2,55 & 2,39 & 3,87 \\
\hline Total & 49,01 & 36,62 & 41,56 & 36,66 & 38,31 & 37,52 & 36,28 & 44,35 \\
\hline Media & $4,08^{\mathrm{a}}$ & $3,05^{\mathrm{b}}$ & $3,46^{\mathrm{a}}$ & $3,06^{\mathrm{a}}$ & $3,19^{\mathrm{a}}$ & $3,13^{\mathrm{a}}$ & $3,02^{\mathrm{a}}$ & $4,03^{b}$ \\
\hline Desviación estándar & 0,52 & 0,37 & 0,68 & 0,59 & 0,62 & 0,77 & 0,58 & 0,84 \\
\hline (ANOVA) Estadístico F & \multicolumn{2}{|c|}{0,49} & \multicolumn{2}{|c|}{1,38} & \multicolumn{2}{|c|}{1,67} & \multicolumn{2}{|c|}{0,41} \\
\hline p-valor & \multicolumn{2}{|c|}{$\mathrm{p}=0,001<0,05$} & \multicolumn{2}{|c|}{$\mathrm{p}=0,28>0,05$} & \multicolumn{2}{|c|}{$\mathrm{p}=0,23>0,05$} & \multicolumn{2}{|c|}{$\mathrm{p}=0,004<0,05$} \\
\hline
\end{tabular}

**Letras distintas en una misma columna indican diferencias estadísticamente significativas $(p<0,05)$,

En general según las percepciones de los estudiantes encuestados, en las áreas de Ingeniería de Alimentos, Ingeniería Química, e Ingeniería de Sistemas, los mayores promedios en las calificaciones $(4,08 \pm 0,52 ; 3,46 \pm 0,68$; y $3,19 \pm 0,62$ respectivamente) lo obtuvieron los profesores del grupo A. Mientras que en Ingeniería Civil los profesores del grupo B fueron los de promedios más altos $(4,03 \pm 0,84)$. Se presentaron diferencias estadísticamente significativas entre los promedios de las calificaciones asignadas a los dos grupos de profesores en las áreas de Ingeniería de Alimentos e Ingeniería Civil $(p<0,05)$. Mientras 
que en Ingeniería Química y Sistemas, no hubo diferencias estadística entre los grupos de profesores $(p>0,05)$. Se observó que en general las calificaciones para ambos grupos de profesores estuvieron entre bueno y aceptable.

\section{CONCLUSIONES}

Los profesores de mayor productividad académica fueron los del área de Ingeniería de Alimentos, seguidos por las áreas de ingeniería Química, Sistemas y por último Ingeniería Civil. No hubo diferencias estadísticas entre las evaluaciones realizadas por los estudiantes, sobre la calidad de enseñanza y la productividad académica de los profesores en las áreas de Ingeniería Química y Sistemas. Se encontró que los estudiantes relacionaron la mayor productividad académica de los profesores de planta, con la percepción de calidad en la enseñanza en Ingeniería de Alimentos, con excepción de Ingeniería Civil, dónde la calificación de los estudiantes no estuvo relacionada con la productividad académica de los profesores. Es necesario generar planes de motivación orientados a los profesores para que estos divulguen sus resultados de investigación en revistas indizadas de índole nacional e internacional, y de esta manera se promueva la investigación en el área de Ingeniería.

\section{REFERENCIAS}

Aguilar, A., A. Carbonell, y E. Cisneros-Cohernour, La evaluación de la docencia en dos universidades públicas latinoamericanas: Cuba y México, Rev. Iberoam.Evaluación Educativa: 7(2), 65-73 (2014)

Alzate-Granados, J.P. y otros cuatro autores, Participation of undergraduate medicine students in medical and academic Colombian journals indexed in Publindex, categories A1 and A2, in the period 2009-2012: a systematic review of the literature, Revista de la Facultad de Medicina: 62(1) 9-15 (2014)

Amado-Moreno, M., J. Sevilla-García y J. Galaz-Fontes, La productividad académica en las instituciones públicas tecnológicas mexicanas, Revista Electrónica Actualidades Inv. en Educ.: 11(4), 125-135 (2013)

Araneda, C., E. Rodríguez y L. Pedraja, Relación entre el Financiamiento Fiscal, la Calidad del Cuerpo Académico y la Retención de Estudiantes Universitarios en Chile, Form. Univ.a: 6(6), 55-64 (2013)

Bolívar, A. y R. Bolívar, Docencia e investigación en el contexto de la universidad actual, Espaço Pedagógico:21(2) 380-402 (2014)

Carrillo-Vargas, E. Academic production of the college teachers and its relationship with the communicative competence, Atenas Revista Científica de Psicología: 4(28) 75-88 (2014)

Comas-Rodríguez O. y A. Rivera-Morales, La docencia universitaria frente a los estímulos económicos, Educação, Sociedade \& Culturas: 32(1) 41-54 (2011)

Concepción-González, V. y H. Ramos-González, Publicaciones científicas de los profesores de la Facultad de Medicina de Villa Clara (2011-2013), Revista Edumecentro: 7(1) 106-119 (2015)

Fassbender, D.S y J.A Silva, ¿Pago por rendimiento? Una discusión de las políticas de incentivación de la productividad académica en Colombia y Alemania. Revista PAPELES, 6(11) 10-19 (2014)

García-Cepero, M. El estudio de productividad académica de profesores universitarios a través de Análisis Factorial Confirmatorio: caso de psicología en Estados Unidos de América, Universitas Psychologica: 9(1), 13-26 (2010)

Gómez-Campo, V. y J.E. Celis-Giraldo, Docencia, estatus, distinción y remuneración, Nómadas: 27(1) 98109 (2007)

Hernández, I. El docente investigador en la formación de profesionales, Revista Virtual Universidad Católica del Norte: 1(27) 1-21 (2011)

Lee, I. Publish or perish the myth and reality of academic publishing, Language Teaching, 47, 250-261 (2014)

López, P.L., Población muestra y muestreo, Punto Cero, 69-74 (2004)

Narváez S. y J. Tovar, La productividad investigativa del docente universitario, Revista de Ciencias Humanas: 18(7) 116-140 (2011)

Uluocha, A. y I. Mabawonku, Legal Information Resources Availability and Utilisation as Determinants of Law Lecturers Research Productivity in Nigerian universities, Inf. \& Knowledge Management: 4(9) 50-58 (2014)

Zavala-González, M. y otros cuatro autores, Productividad científica de los profesores-investigadores de la maestría en educación de la Universidad Popular de la Chontalpa: un diagnóstico situacional, Perspectivas profesores: 49 (3) 21-28 (2012) 